\title{
Aplicación del modelo de clase inversa en el campo de la Ingeniería Térmica: análisis y comparación de varios contextos ${ }^{1}$
}

\author{
Begoña Peña ${ }^{a}$, I. Zabalza ${ }^{b}$, E. Llera ${ }^{c}$, S. Usón ${ }^{d}$, L.M. Romeo
}

Universidad de Zaragoza - Departamento de Ingeniería Mecánica (España)

abpp@unizar.es, bizabal@unizar.es, ellera@unizar.es, ${ }^{\mathrm{d}}$ suson@unizar.es, ${ }^{\mathrm{e}}$ luismi@unizar.es.

\begin{abstract}
The flipped classroom model (FCM) presents many advantages with respect to the traditional model, as the students play a leading role in attendance sessions increasing their motivation and satisfaction, active methodologies and Information and Communication Technologies (ICT) are naturally integrated into the learning process, and certain transverse competencies can be developed and evaluated in the classroom.

Motivated by these advantages, the authors have carried out various experiences in FCM during the last two academic courses in the context of Thermal Engineering at the University of Zaragoza (Spain). Under the framework of two projects financed by the Program for Promoting Teaching Innovation (PIIDUZ_16_032 and PIIDUZ_17_299), different methodologies and resources have been implemented for in-class and home activities.

The observations and results of these experiences in different subjects and academic levels are here analyzed and compared with respect to various criteria. In all cases, the reception from the students was very positive, but the influence on the learning process depends in large extent on the use that students make of the materials provided by the teacher. Some aspects to this respect are discussed in the paper.
\end{abstract}

Keywords: Moodle, Educational Videos, Flipped Classroom, University Innovation, Engineering Education.

\section{Resumen}

El modelo de clase inversa (FCM) presenta muchas ventajas con respecto al modelo tradicional, ya que los estudiantes desempeñan un papel principal en las sesiones presenciales aumentando esto su motivación y satisfacción, las metodologías activas y las Tecnologías de la Información y la Comunicación (TIC) se integran naturalmente en el proceso de aprendizaje, y ciertas competencias transversales pueden ser desarrolladas y evaluadas en el aula.

Motivados por estas ventajas, los autores han llevado a cabo varias experiencias en FCM durante los dos últimos cursos académicos en el contexto de la Ingeniería Térmica en la Universidad de Zaragoza (España).

\footnotetext{
${ }^{1}$ Este trabajo se ha desarrollado dentro de los proyectos de innovación docente PIIDUZ_17_299 y PRAUZ_17_002 (Programa de Proyectos de Innovación Docente para Grupos de Profesores del Vicerrectorado de Política Académica de la Universidad de Zaragoza).
} 
Uso de recursos y rendimiento en las actividades evaluación: análisis y comparación de resultados en el Grado en Ingeniería de Tecnologías Industriales

En el marco de dos proyectos financiados por el Programa de Incentivación de la Innovación Docente (PIIDUZ_16_032 y PIIDUZ_17_299), se implementaron diferentes metodologías y recursos para las actividades a realizar dentro y fuera del aula.

Las observaciones y los resultados de estas experiencias en diferentes materias y niveles académicos se analizan aquí y se comparan con respecto a varios criterios. En todos los casos, la recepción de los estudiantes fue muy positiva, pero la influencia en el proceso de aprendizaje depende en gran medida del uso que los estudiantes hacen de los materiales proporcionados por el profesor. Algunas cuestiones a este respecto se discuten en el presente documento.

Palabras clave: Moodle, Vídeos Didácticos, Clase Inversa, Innovación Universitaria, Educación en Ingeniería.

\section{Introducción}

El Espacio Europeo de Educación Superior (EEES) y los nuevos retos planteados por la sociedad actual están impulsando una profunda revisión de los modelos de enseñanzaaprendizaje y supondrán en el futuro un cambio sustancial en los roles que juegan profesor y alumno. En este sentido, el modelo pedagógico conocido como clase inversa (flipped classroom model, FCM) presenta muchas ventajas (Bergmann 2012) frente al modelo tradicional de clase centrada en la transferencia de información del profesor al alumno (Figura 1).

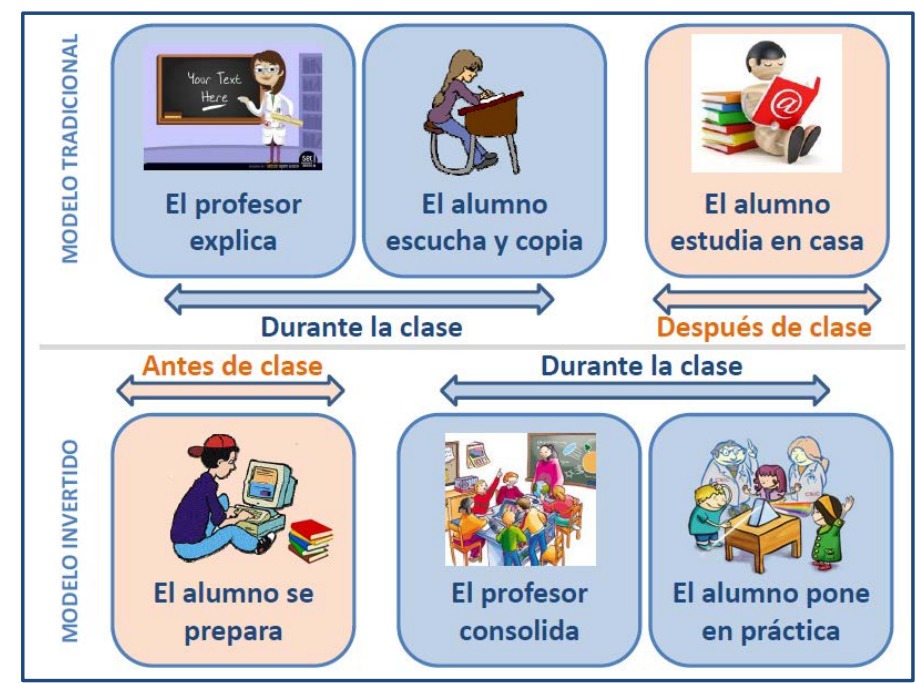

Fig.1 Comparación de la distribución de actividades en el modelo tradición y el modelo de clase inversa (elaboración propia, (Varios, 2018))

(cc) EY-NC-ND 2018, Universitat Politècnica de València

Congreso IN-RED (2018) 
Por un lado, el modelo FCM permite implantar en el aula metodologías activas, como el aprendizaje basado en problemas, la simulación, los debates o la metodología del puzzle, que han demostrado ser muy eficaces para conseguir un aprendizaje profundo.

Estas metodologías estimulan los niveles superiores de razonamiento de la taxonomía de Bloom (Anderson, 2001) que tan necesarios son para los titulados universitarios, pero requieren un tiempo en el aula que con el modelo tradicional no está disponible. Bajo el modelo de clase inversa, la transferencia de información que ocuparía prácticamente todo el tiempo en la clase magistral se traslada mediante diversos recursos a un momento previo en el que el alumno prepara la clase. De esta forma, aunque el profesor explique ciertos conceptos y resuelva dudas durante la clase, quedará tiempo suficiente para que los alumnos practiquen lo que han aprendido con metodologías, actividades y recursos que serán seleccionados por el profesor en función de cada materia y del nivel académico.

En este entorno, las TIC se integran de manera natural en el proceso de aprendizaje, contribuyendo a una mayor participación del alumnado, a través de herramientas como Kahoot! o Socrative. En la clase tradicional, los alumnos suelen mostrarse poco participativos, especialmente si el grupo es numeroso, incluso cuando el profesor dinamiza la clase con preguntas o ejercicios. Si la clase no se centra en las explicaciones del profesor, el alumno se siente más relajado para plantear sus dudas, responder a preguntas o discutir sobre la materia estudiada.

Además, el modelo FCM favorece el desarrollo y facilita la evaluación de competencias trasversales, como el trabajo en grupo, la autonomía o aprender a aprender, que tan buscadas son por los empleadores entre los titulados universitarios. Todo esto hace que el alumno cobre protagonismo en las clases presenciales, aumentando su grado de motivación y de satisfacción y permitiendo una mejor gestión de la diversidad por parte del profesor (Figura 2).

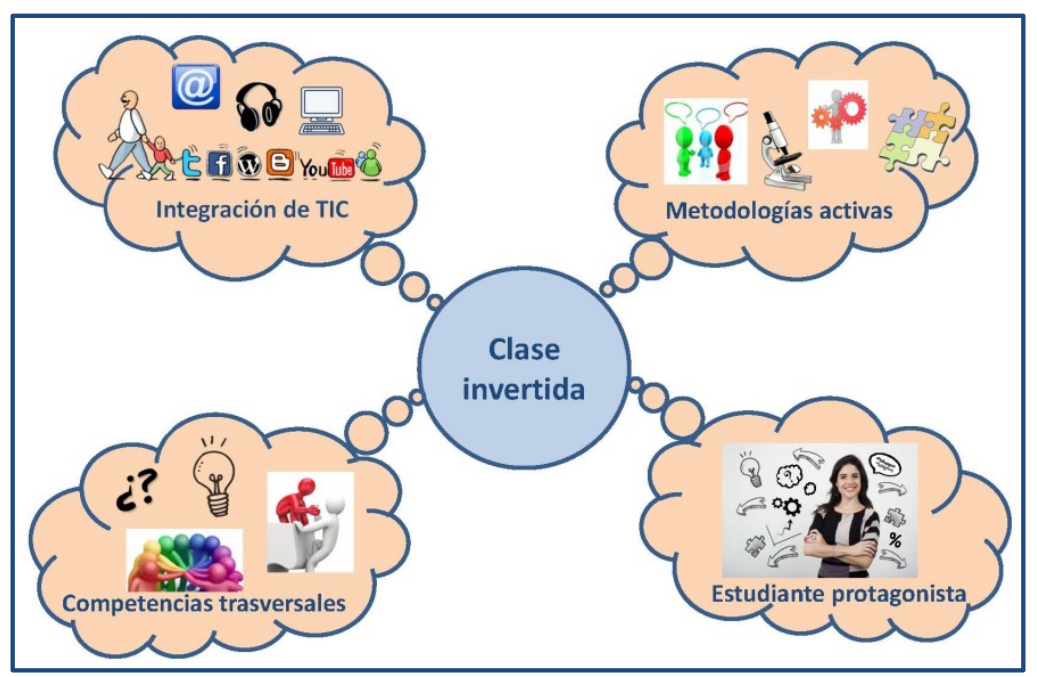

Fig.2 Ventajas del modedo de clase inversa (elaboración propia, (Varios, 2018)). 
De acuerdo con esto, el rol del profesor cambia, pero sigue siendo esencial en el aprendizaje del estudiante para seleccionar los recursos y metodologías de trabajo, para planificar, guiar y servir de apoyo en las actividades de aprendizaje y para evaluar tanto los resultados alcanzados por el estudiante, como el éxito del proceso en sí (Tourón, 2015).

Este modelo se ha ido adoptando primero en las escuelas de Primaria y Secundaria, para más recientemente llegar a la Universidad. El modelo de clase inversa se está implantando en los mejores centros de enseñanza del mundo, como el Shireland Collegiate Academy (Inglaterra), la Universidad de Harvard o el Instituto Tecnológico de Massachusetts (MIT). En España, la Universidad Politécnica de Valencia es pionera en dos líneas de trabajo: implantar el modelo de clase inversa en cursos completos de varias titulaciones y en desarrollar un procedimiento a nivel institucional para la evaluación de las competencias trasversales y su inclusión en el expediente académico de cada estudiante (UPV, 2017). El trabajo que aquí se presenta pretende sumarse a estas iniciativas con el objetivo fundamental de aprovechar al máximo el tiempo que alumno pasa en clase y en última instancia mejorar los resultados de aprendizaje y la adquisición de competencias.

La idea nace de la experiencia docente de los autores en varias titulaciones impartidas en la Escuela de Ingeniería y Arquitectura (EINA) de la Universidad de Zaragoza y de la demanda continua por parte de los alumnos de hacer las clases más participativas y sobre todo más prácticas. Adicionalmente, el tiempo dedicado a ciertas materias del área de Máquinas y Motores Térmicos se ha visto reducido en los nuevos planes de estudio, sin que hayan cambiado las competencias que deben adquirir los alumnos. Ante este reto, es preciso aprovechar al máximo el tiempo en el aula y se ha apostado por el modelo de clase inversa en el marco de dos proyectos financiados por el Programa de Incentivación de la Innovación Docente (PIIDUZ_16_032 y PIIDUZ_17_299).

En este trabajo se recogen las metodologías, las observaciones y los resultados de varias experiencias en diferentes materias y niveles académicos y se comparan con respecto a varios criterios. Finalmente, se extraen los aspectos positivos y aquellos en los que se puede mejorar o en los que se deben implementar acciones correctivas para superar los problemas detectados hasta ahora.

\section{Objetivos}

El objetivo de este trabajo es comparar las experiencias que han desarrollado los autores sobre FCM en los dos últimos cursos en diferentes asignaturas y titulaciones, con el fin de difundir las prácticas que han resultado positivas para que se puedan repetir en otros ámbitos. También se recogen los problemas encontrados en algunos casos para su implantación y sugerencias para mejorar su eficacia, de manera que nuestra experiencia sirva a otros profesores para el diseño de sus propias materias.

Los objetivos específicos de este trabajo son:

- Recopilar los datos de las diversas experiencias: observaciones, encuestas.

- Comparar experiencias enfocadas al mismo tipo de clase: magistral o prácticas.

(cc) EY-NC-ND 2018, Universitat Politècnica de València

Congreso IN-RED (2018) 
- Comparar la información en base a dos criterios: nivel de estudios (grado/máster) y perfil profesional de la titulación (mecánico, químico, industrial).

- Recopilar las buenas prácticas, los problemas encontrados y estrategias propuestas para mejorar en el futuro.

\section{Desarrollo de la innovación}

El presente estudio se ha llevado a cabo con alumnos de diferentes titulaciones impartidas en la EINA (Universidad de Zaragoza). Las asignaturas se han elegido en base a la experiencia previa de los profesores, tanto en aula invertida como en la impartición de las propias asignaturas. Con respecto a la materia concreta en la que se aplicará el modelo de aula inversa, se ha tenido en cuenta que tenga un carácter esencialmente aplicado, que no entrañe conceptos complejos que dificulten el aprendizaje autónomo y la disponibilidad de materiales ya desarrollados propios y ajenos. En la Tabla 1 se resume la información básica de las experiencias en FCM analizadas en este documento.

Tabla 1. Datos generales de las experiencias en FCM.

\begin{tabular}{|c|c|c|c|c|}
\hline Asignatura & Matriculados & Horas & $\begin{array}{l}\text { Titulación } \\
\text { / Semestre }\end{array}$ & Curso \\
\hline Termodinámica Técnica y Fund. & 70 & 6 & CITI S & 2016-2017 \\
\hline Transmisión de Calor & 52 & 6 & Gl11 S3 & 2017-2018 \\
\hline Ingeniería Térmica & 51 & 5 & GITI S4 & 2016-2017 \\
\hline Termotecnia & 32 & 3 & GIQ S6 & 2016-2017 \\
\hline Máquinas y Motores Térmicos & 106 & 3 & GIM S6 & 2016-2017 \\
\hline $\begin{array}{l}\text { Eficiencia Energética en la } \\
\text { Edificación }\end{array}$ & 8 & 2 & MUERyEE & 2016-2017 \\
\hline
\end{tabular}

Todas las asignaturas son de 6 ECTS y obligatorias de grado, excepto la asignatura de Eficiencia Energética en la Edificación que es optativa de 5 ECTS en el Máster Universitario de Energías Renovables y Eficiencia Energética (MUERyEE). La nomenclatura utilizada en la tabla para los diferentes grados es la siguiente: GITI - Grado en Ingeniería de Tecnologías Industriales, GIQ - Grado en Ingeniería Química, GIM Grado en Ingeniería Mecánica. En los siguientes epígrafes se describe brevemente la metodología de trabajo seguida en cada asignatura.

\subsection{Termodinámica Técnica y Fundamentos de Transmisión de Calor}

Esta asignatura es la base de todas las asignaturas posteriores de la rama energética y, por lo tanto, esencial para la titulación. La metodología seguida se ha adaptado a cada lección particular. El modelo FCM se ha aplicado principalmente en la lección completa que corresponde a los procedimientos para el cálculo de propiedades de sustancias puras y en ciertas sesiones de los temas de ciclos termodinámicos, por ser temas esenciales para la 
asignatura y además disponer de videos explicativos suficientes que se proporcionan para cada sesión junto con otros materiales a través de Moodle (Peña, 2017-2018). Las sesiones presenciales se dedican a resolver dudas y problemas en pequeños grupos.

En las prácticas también se ha seguido este modelo con el fin de solventar el desfase que hay con respecto a las clases de teoría: a través de Moodle se proporcionan con antelación una serie de materiales (guion, apuntes, videos) para que el estudiante prepare la práctica y resuelve un cuestionario que entrega antes de la práctica. En la sesión, el profesor realiza un breve resumen, resuelve las dudas y deja el resto del tiempo para que los estudiantes por parejas reuelvan un problema más complejo, cuyos resultados entregan al final de la sesión.

Para completar la experiencia en FCM se realizan encuestas antes y después para valorar el nivel de satisfacción de los estudiantes.

\subsection{Ingeniería Térmica}

Esta asignatura se imparte también en el segundo curso pero en el semestre siguiente a la asignatura explicada en el punto anterior. Los contenidos son esencialmente complementarios, aunque ciertas definiciones y conceptos son los explicados en la asignatura de Termodinámica Técnica. El grupo es el mismo y, por lo tanto, salvo repetidores los alumnos son los mismos. Dadas las fechas de la presente publicación, sólo se dispone de la información correspondiente al curso 2016-2017.

Para la implementación de FCM se eligió el tema de Intercambiadores de Calor, por ser un tema eminentemente de práctico. Con antelación, se desarrollaron los videos necesarios y se distribuyeron a través de Moodle, junto con bibliografía para preparar cada una de las sesiones. Una vez en el aula, se realiza un repaso general del contenido de los videos y se remarcan las ideas más importantes. Tras resolver las dudas, los estudiantes resuelven diversas cuestiones y problemas.

\subsection{Termotecnia}

La asignatura de Termotecnia (semestre S6) es continuación natural de la asignatura de Termodinámica Técnica y Fundamentos de Transmisión de Calor (semestre S4), en la que se explican los mecanismos básicos y se realiza una introducción a la conducción del calor. Para la experiencia en FCM, se ha seleccionado el tema de Conducción en Problemas Unidimensionales, que es un tema ya estudiado en una asignatura previa al que se le puede dedicar poco tiempo, pero que, en general, los alumnos no recuerdan.

Con antelación suficiente, se informa a los estudiantes de cómo y cuándo se realizará la actividad y se cuelgan en Moodle los recursos necesarios para que el estudiante repase la materia correspondiente (apuntes y ejemplos resueltos). El día de la sesión presencial, se realiza un breve repaso, se resuelven dudas y a continuación se plantean dos problemas para consolidar el aprendizaje. Al final de la sesión se ponen en común las soluciones.

(cc) EY-NC-ND 2018, Universitat Politècnica de València

Congreso IN-RED (2018) 


\subsection{Máquinas y Motores Térmicos}

Esta asignatura de tercer curso (semestre S6) cierra las materias obligatorias de la rama de energía en el Grado de Ingeniería Mecánica, tras dos asignaturas básicas en las que se estudia Termodinámica Técnica (S2) y Transferencia de Calor (S5). El modelo FCM se ha aplicado a una práctica de simulación sobre Ciclos de Turbina de Vapor, con el programa Engineering Equation Solver (EES).

Parte de sus contenidos deberían ser conocidos por el alumno, pero se sabe de cursos anteriores que no los tienen bien asimilados o los han olvidado debido el tiempo transcurrido desde su impartición (S2). Por ello, con varias semanas de antelación, se pone a disposición del estudiante el material necesario: guion, video específico de ciclos de turbina de vapor y un archivo EES con el modelo semiresuelto. Durante la primera hora de la sesión se repasan los aspectos clave, se plantean preguntas para comprobar que se han cubierto los objetivos tras ver el video y se resuelven las dudas que hayan surgido. En las siguientes dos horas el estudiante completa el modelo con ayuda del profesor y entrega el informe de resultados al finalizar la práctica.

\subsection{Eficiencia Energética en la Edificación}

Esta asignatura constituye la única experiencia realizada en estudios de Máster y en una asignatura optativa. A la diferencia existente en el nivel académico y en la motivación con respecto las experiencias anteriores, hay que añadir que el número de alumnos matriculados es mucho menor. Todo esto plantea una situación a priori muy diferente.

El tema seleccionado ha sido la Arquitectura bioclimática y estrategias pasivas de ahorro energético en edificios. Para la preparación de la materia se les proporciona documentación en formato texto vía Moodle y bibliografía específica. En clase se plantea un cuestionario con más de 20 preguntas para evaluar el aprendizaje sobre el tema y para abrir una discusión entre los alumnos sobre temas concretos, que resuelva dudas que a priori no se habían planteado. En principio, cada pregunta se asigna a un estudiante concreto, para después dar el turno a los demás con la moderación del profesor.

$\mathrm{Al}$ final de la clase, se realizan dos encuestas para conocer su opinión sobre las clases universitarias y sobre su satisfacción con respecto al modelo FCM.

\section{Resultados}

Con el fin de documentar las observaciones de los profesores de una forma sistemática, se han desarrollado dos formularios: uno en el que registrar la información específica de cada sesión (Figura 3) y otro en el que se resumen las observaciones generales de la experiencia y la metodología seguida en la misma (Figura 4). Se trata de primeras versiones que se irán completando en función de las deficiencias observadas o de que se planteen nuevos estudios comparativos. 


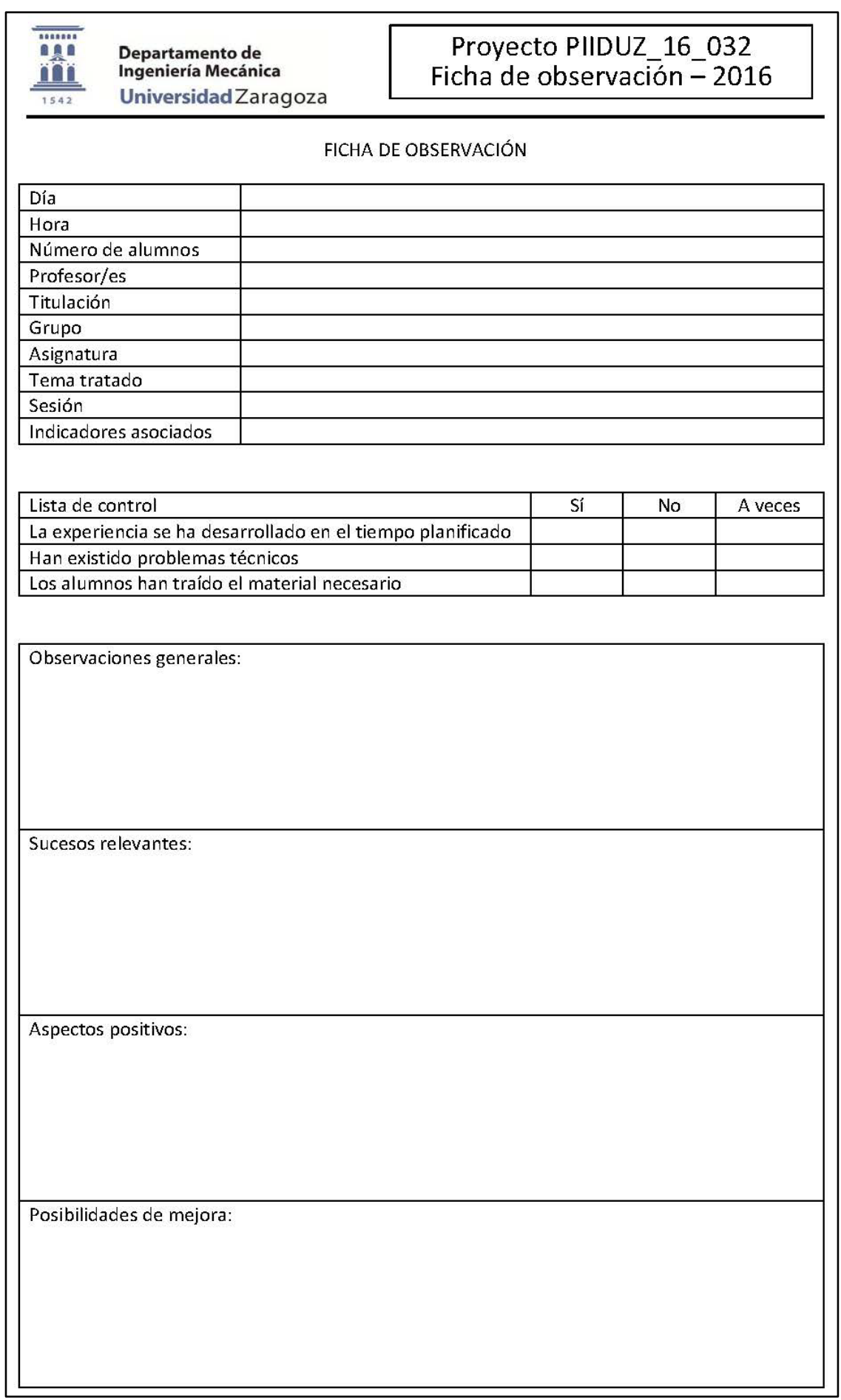

Fig.3 Ficha de observación a completar por el profesor en cada sesión de FCM.

(cc) EY-NC-ND 2018, Universitat Politècnica de València

Congreso IN-RED (2018) 


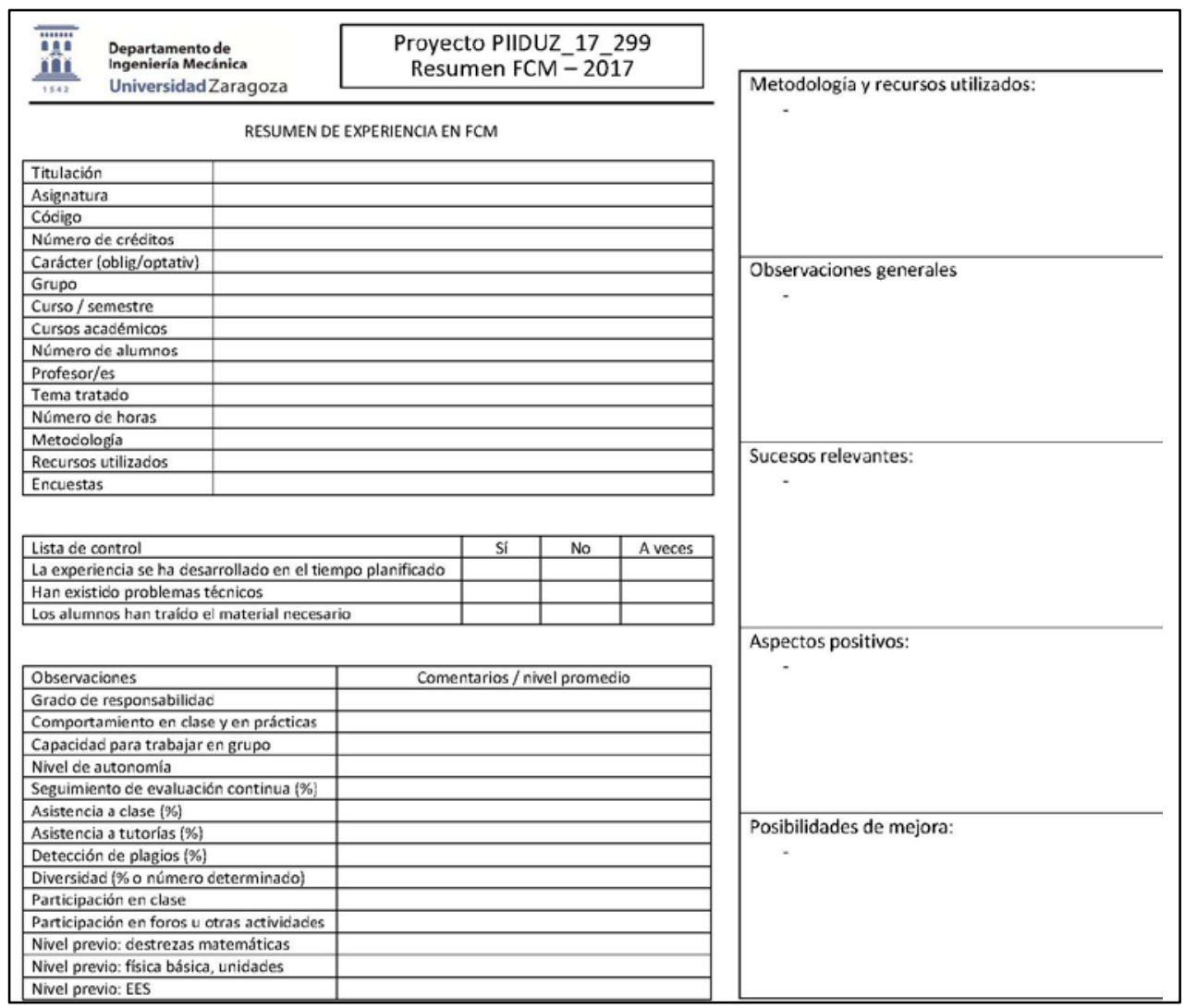

Fig.4 Plantilla a completar por cada experiencia en FCM a modo de resumen.

Asimismo se han desarrollado dos encuestas para los estudiantes: una sobre su experiencia personal sobre los modelos y metodologías utilizados en las clases universitarias y otra especifica de la experiencia en FCM, con el fin de evaluar su grado de satisfacción (Peña, 2017). Además, la evaluación de cada video docente se ha realizado a través de una encuesta breve de Moodle con sólo cinco preguntas (escala de Likert) para promover la participación (Zabalza, 2017-2018). La comparativa que se presenta en los siguientes apartados se ha realizado en base a la información recogida en esos formularios y encuestas.

\subsection{Experiencias orientadas a las sesiones de prácticas}

Uno de los problemas abordados mediante el modelo de clase inversa ha sido la desincronización temporal entre las clases magistrales y las prácticas de laboratorio, que en muchas ocasiones no se puede evitar por la rigidez del calendario oficial. Como se ha explicado en los epígrafes 2.1 y 2.4, se ha aplicado una metodología similar en las asignaturas de Termodinámica Técnica (TT) y de Máquinas y Motores Térmicos (MMT): proporcionar materiales (textos, videos) con antelación para preparar la práctica, reducir el tiempo dedicado a la explicación y dejar más tiempo para que el alumno resuelva el caso planteado. En TT se ha aplicado este modelo durante los tres últimos cursos académicos, mientras que en MMT sólo se dispone de los datos del curso 2016-2017.

(c) ) EY-NC-ND 2018, Universitat Politècnica de València 
Para los cursos 2015-2016 y 2016-2017, en ambas asignaturas se comprueba en Moodle que sólo alrededor del $50 \%$ de los alumnos había visto el video con antelación y de hecho en GIM se detectó que algunos estudiantes estaban viendo el video durante la sesión. Además, pocos alumnos responden correctamente a las preguntas de control que el profesor hace mientras repasa los conceptos más importantes, lo que demuestra que los alumnos dedican poco (o nada) de tiempo a preparar la práctica.

En MMT se ha propuesto preparar un cuestionario evaluable previo para incertivar el trabajo previo. Sin embargo, esta medida no ha resultado efectiva en la asignatura de TT, en la cual, habiéndose detectado este problema en cursos anteriores, se vienen utilizando cuestionarios desde el inicio del grado en 2011. Hasta el presente curso se entregaban en papel y no tenían un peso definido en la nota final. Ahora se han implementado en Moodle y cuentan 1 punto de la nota de prácticas. La proporción de alumnos que ha visto el video ha subido hasta el $60 \%$ y la nota de esos cuestionarios ha mejorado desde una media de 7 hasta una media de 9 puntos. Sin embargo, no se ha observado una mejora significativa en la preparación de los estudiantes para resolver el problema de simulación. Por tanto, el aumento podría deberse a una mayor facilidad para ‘copiar’ y no a un mayor interés.

Por esta razón, se está planteando la posibilidad de que resuelvan un cuestionario de autoevaluación sobre el video asociado a cada práctica, bien integrado en el propio video, bien en Moodle, y que el cuestionario de evaluación se realice durante los primeros 10 minutos de la sesión y sea obligatorio alcanzar una nota mínima para poder realizar la práctica. En caso de no superarse, el estudiante debería volver otro día para una sesión específica de recuperación.

En ambas asignaturas se ha observado que los alumnos, en general, tienen poca iniciativa, baja capacidad de reflexión y poca autonomía para abordar problemas nuevos. Esto creemos que se debe a que el modelo tradicional de enseñanza-aprendizaje no favorece estas competencias. Por esta razón, se está tratando de implementar FCM en estas mismas asignaturas también en las clases magistrales. Sin embargo, el problema trasciende a toda la titulación y deberían adoptarse medidas globales para atajarlo.

\subsection{Experiencias orientadas a las sesiones magistrales}

Aquí se van a comparar las observaciones en dos asignaturas para el mismo curso y grupo del grado GITI (Termodinámica Técnica e Ingeniería Térmica) y la asignatura de Termotecnia en GIQ. En los tres casos se ha seguido un procedimiento similar: proporcionar videos y textos para preparar la materia, resolver dudas al comienzo de la clase y dejar que los alumnos resuelvan varios ejercicios en grupo y con ayuda del profesor.

En todos los casos, la mayor cercanía del profesor favorece la interacción y que los alumnos planteen abiertamente sus dudas. Tanto es así que hay muchos momentos en que varios alumnos tienen que esperar su turno. Se planteó ya el curso pasado la conveniencia de que haya apoyo de un segundo profesor en el aula y esto se ha aplicado durante el presente curso en TT, siendo una solución que ha tenido buena acogida entre los estudiantes.

(cc) EY-NC-ND 2018, Universitat Politècnica de València 
En todos los casos, se considera que los objetivos mínimos de aprendizaje se han cumplido, pero también es cierto que a la mayoría de los estudiantes no les da tiempo de completar las tareas encomendadas para cada clase, especialmente en las primeras sesiones en que están menos habituados. Por esta razón, es muy importante que el profesor planifique cuidadosamente las tareas que el alumno debe abordar y las priorice guiando a los alumnos hacia los apartados más importantes, dejando para casa lo que es de repaso o repetitivo.

\subsection{Diferencias entre grados de distinto perfil profesional}

El comportamiento ha sido diferente entre grados. En GITI, un buen número de alumnos no ha traído el material necesario el primer día y tardan bastante en ponerse a trabajar en la primera sesión. Esta actitud cambia en las siguientes sesiones que son mucho más provechosas, con buen ambiente de trabajo y con un porcentaje de alumnos pasivos que va decreciendo progresivamente. En Ingeniería Térmica, incluso se observó un aumento de la asistencia desde un $60 \%$ hasta casi un $90 \%$. Como se ha explicado antes, por tratarse del mismo grupo de alumnos que para Termodinámica Técnica, la mayoría conocían el procedimiento de la clase inversa y han podido estar más motivados para asistir.

Se han observado diferencias reseñables en la actitud de los estudiantes con respecto a Termotencia de GIM. En este grupo, parecen tener un mayor grado de responsabilidad y compromiso. La gran mayoría viene a las sesiones de FCM con los materiales necesarios y se ponen a trabajar de manera inmediata. Lo más llamativo es que, en general, prefieren trabajar de forma individual y contrastar con el profesor o con sus compañeros los resultados finales. Hay que destacar que en el grado GIQ existe una proporción del 45\% de estudiantes de género femenino frente al $20 \%$ de GITI. Consideramos que sería interesante analizar la influencia de este hecho en los hábitos y actitudes de los alumnos desde un punto de vista global a nivel universitario.

\subsection{Comparativa entre diferentes niveles educativos}

Como se recoge en la Tabla 1, sólo se ha realizado una experiencia de FCM en máster y, además, ésta corresponde al único caso en que la asignatura es optativa y el grupo es mucho menos numeroso que para las asignaturas de grado. En general, se observa un mayor grado de responsabilidad y de motivación y una mejor preparación académica para abordar la asignatura. Esto se debe, obviamente, a un mayor grado de madurez y al hecho de ser una asignatura no obligatoria, cuya matrícula indica interés en la materia.

La metodología seguida para la preparación de las clases es similar a la usada en las asignaturas de grado: dejar documentación y bibliografía específica en la plataforma Moodle. Por el contrario, se realiza una actividad diferente en la clase presencial: un cuestionario de más de 20 preguntas clave obliga a que los alumnos razonen y expliquen la respuesta a los compañeros. Además, el carácter abierto de la respuesta de algunas de las preguntas da pie a que se realice un pequeño debate sobre las mejores soluciones en cada caso.

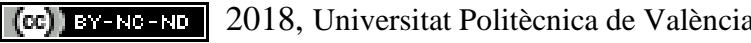

Congreso In-Red (2018) 
A pesar de todas estas diferencias, la actitud de los alumnos no difiere demasiado de la observada en los grados. La mayoría de los estudiantes responden a las preguntas más en base al sentido común y a conocimientos de asignaturas básicas, que razonando con argumentos específicos de la materia que deberían haber preparado con los recursos proporcionados para la sesión. De nuevo, se observa que, aunque la participación en el debate es alta y tratan de responder correctamente y de comprender las explicaciones a cada pregunta, los estudiantes dedican poco esfuerzo a preparar las clases como ocurre también en los grados.

Con respecto a su opinión sobre este tipo de modelo pedagógico y a las clases universitarias en general, se obtienen curiosamente resultados muy parecidos a los obtenidos en el grado GITI (Peña, 2017). La mayor demanda es la misma: que las clases sean más prácticas y que el profesor resuelva más problemas. En ambos estudios consideran que el modelo tradicional se sigue en más de un $80 \%$ de las clases. El nivel de asistencia también ronda el $80 \%$ entre los encuestados, aunque hay que decir que solo alrededor del $75 \%$ de los matriculados en ambos casos estaba en el aula para hacer la encuesta. La mayor diferencia está en que más del $80 \%$ los alumnos encuestados del máster consideran que el grado de aprovechamiento en las clases tradicionales es alto o muy alto, frente al 27\% de GITI.

Con respecto a la experiencia en FCM las opiniones están repartidas en torno al 50\% entre el modelo tradicional y el aula invertida, tanto en grado como en máster. En cuanto a los materiales, un 50\% de los estudiantes de GITI consideraron los videos de gran utilidad frente al 20\% de los alumnos del máster. En cualquier caso, una buena proporción (35\% en GITI y $80 \%$ en MUERyEE) reconoce que, aunque los considera interesantes, no les ha dedicado mucho tiempo. En este sentido, parece necesario insistir en que es imprescindible estudiar esos materiales para aprovechar las horas presenciales y concentrarlos al máximo para que su extensión no desmotive al alumnado. Además, hay que tener en cuenta factores externos a la asignatura, tales como actividades de otras asignaturas y vacaciones o festividades, que lejos de motivar al estudio reducen la dedicación a este tipo de tareas.

En cuanto al grado de satisfacción con respecto al modelo FCM, fue alto o muy alto para el $30 \%$ de GITI y para el 65\% de MUERyEE. Sin embargo, el grado de aprovechamiento en opinión de los estudiantes fue alto o muy alto para un 50\% en GITI, mientras que sólo lo fue para el 20\% de los estudiantes de MUERyEE. Esto puede deberse a que los alumnos no dedicaron el tiempo suficiente a los materiales colgados en Moodle, como así lo reconoce el $65 \%$ de los alumnos. En cualquier caso, será necesario investigar con mayor detalle el por qué de estos resultados en la asignatura de Eficiencia Energética para mejorar en los próximos cursos. 


\section{Conclusiones}

En este trabajo se han analizado y comparado cinco experiencias sobre clase inversa en diferentes asignaturas, titulaciones y niveles académicos. En todos los casos, el profesor considera que es un método eficaz, que habrá que seguir mejorando, y que en general ha tenido buena acogida por parte de los estudiantes. Las encuestas y las fichas de observación han demostrado ser herramientas necesarias para la evaluación del modelo pedagógico y para mejorar curso a curso.

Las principales conclusiones que se extraen de este estudio son las siguientes:

- Los materiales para preparar las clases deben ser muy específicos del tema a tratar, con una extensión lo más reducida posible para esto no desmotive al alumno. En este sentido, causa mejor imagen que estén desarrollados por el profesor y que no sean textos genéricos de la asignatura.

- También es motivador que entre los recursos proporcionados para preparar la práctica exista algún tipo de tarea en la que apliquen lo aprendido. Esto servirá de herramienta de control para que el profesor compruebe que han estudiado y para que el alumno se autoevalúe y vuelva sobre aquellos conceptos que no tiene claro.

- Aunque no debería ser tarea del profesor, parece ser muy importante recordar con cierta insistencia la necesidad de revisar los materiales antes de la sesión, ya que tanto en grado como en máster los alumnos han reconocido que no han dedicado suficiente tiempo.

- En grupos numerosos se ha constatado la necesidad de contar con dos profesores para atender las dudas de los alumnos, ya que la cercanía del profesor y la actividad práctica crean un ambiente abierto en el que los alumnos se hacen más preguntas y quieren resolver sus dudas.

- En su aplicación a las prácticas de laboratorio, convendrá que los alumnos resuelvan un cuestionario de autoevaluación sobre los recursos asociados a cada práctica y que se realice un cuestionario de evaluación durante los primeros minutos de la sesión en el que sea obligatorio alcanzar una nota mínima para poder realizar la práctica. En caso de no superarse, el estudiante debería volver otro día para realizar la práctica en una sesión específica de recuperación. Tenemos conocimiento de que este procedimiento se aplica con éxito en titulaciones del área científica y médica y pensamos que también resultará eficaz para aumentar el compromiso en nuestras asignaturas. 
Uso de recursos y rendimiento en las actividades evaluación: análisis y comparación de resultados en el Grado en Ingeniería de Tecnologías Industriales

\section{Referencias}

ANDERSON, L.W. et al. (2001). “A Taxonomy for Learning, Teaching, and Assessing: A revision of Bloom's Taxonomy of Educational Objectives”. New York: Pearson, Allyn \& Bacon.

BERGMANN, J. y SAMS, A. (2012) "Flip your classroom: Reach every student in every class every day”, Washington, DC: Internal Society for Technology in Education, 2012.

PEÑA, B. et al. (2017) "Experiencia piloto de aula invertida para mejorar el proceso de enseñanzaaprendizaje en la asignatura de Termodinámica Técnica”. En: Actas del congreso INRED 2017, Valencia, Editorial Universitat Politècnica de València. Doi: 10.4995/INRED2017.2017.6868. Disponible en: < http://ocs.editorial.upv.es/index.php/INRED/INRED2016/paper/view/4430>.

PEÑA, B. (2018) “Defining quantitative and automated rubrics from assessment activities scores” En: INTED18 Proceedings of the $12^{\text {th }}$ annual International Technology, Education and Development Conference, Valencia, IATED Academy, pp. Doi:

TOURÓN, J. y SANTIAGO, R. (2015). “El modelo Flipped Learning y el desarrollo del talento en la escuela”. Revista de Educación, n ${ }^{\circ}$ 368, p. 196-231.

UPV (2017). Flip Teaching. Universidad Politécnica de Valencia. https://www.upv.es/entidades/ETSINF/info/967104normalc.html [Consulta: 19 de enero 2017].

VARIOS (2018). Imágenes de diferentes fuentes: http://www.csicenlaescuela.csic.es/; http://clipground.com/image-post/59640-networking-clipart-11.jpg.html; https:/www.vexels.com; commons.wikimedia.org/wiki/File:Jigsaw.svg; www.flickr.com/photos/96737006@N04/9071059572, http://www.premioscortesdecadiz.es/jovenemprendedor/roberta-medina/. [Consulta 16 de mayo de 2018].

ZABALZA, I., PEÑA, B., LLERA, E.M. y USÓN, S. (2016) "Improving the teaching-learning process using educational videos as reusable learning objects in the field of thermal engineering", Proceedings of the $8^{\text {th }}$ International Conference on Education and New Learning Technologies (EDULEARN 2016), Barcelona, IATED Academy, pp. 363-372. Doi: 10.21125/edulearn.2016.1068.

ZABALZA, I., et al. (2017) "Development of educational videos as reusable learning objects for their integration into an Open Courseware on fundamentals of thermodynamics and thermal engineering", En: INTED17 Proceedings of the $11^{\text {th }}$ annual International Technology, Education and Development Conference, Valencia, IATED Academy, pp. 4453-4461. Doi: 10.21125/inted.2017.1055.

(c) EY-NC-ND 2018, Universitat Politècnica de València 